\title{
OPTIMIZING THE TAKING OF THE SAMPLES FROM THE CONTAMINATED SITES WITH THE AID OF THE DRONES
}

\author{
Dorin EFTIMIE \\ "Dunarea de Jos" University of Galati, Romania \\ e-mail: dorin.eftimie@ugal.ro
}

\begin{abstract}
Monitoring environmental parameters as well as taking samples from contaminated sites using drones is an optimized solution to transmit real-time minimum in-flight data on samples taken.

$3 D$ modeling using NX 8.0 software allowed the modular construction of experimental drone.

The following monitoring tests were performed with the help of the experimental drone:

- video testing of an area on the Braila-Galati dam and capturing the images on digital memory storage.

- collection of samples from water as well as depth measurements.

- monitoring the quality of the air as well as the quantity of pollution in urban traffic at a crossroad in the city of Braila.

The drone can be an efficient solution for taking samples from contaminated sites depending on the equipment with which it is equipped.
\end{abstract}

KEYWORDS: Drone, 3D model, monitoring sites environment parameters

\section{Overview}

Monitoring of environmental parameters may be done with the help of drones.

The technology has developed very rapidly and new, more efficient monitoring methods and reduced costs for timely information collection and communication have emerged.

\section{Drone 3D modeling and the model}

In Figure 1 is shown the 3D modelling of the drone with software NX 8.0 from Siemens. The 3D modelling allowed virtual verification of the correctness of the design [1].

In Figure 2 is shown the drone model and all its components [2].

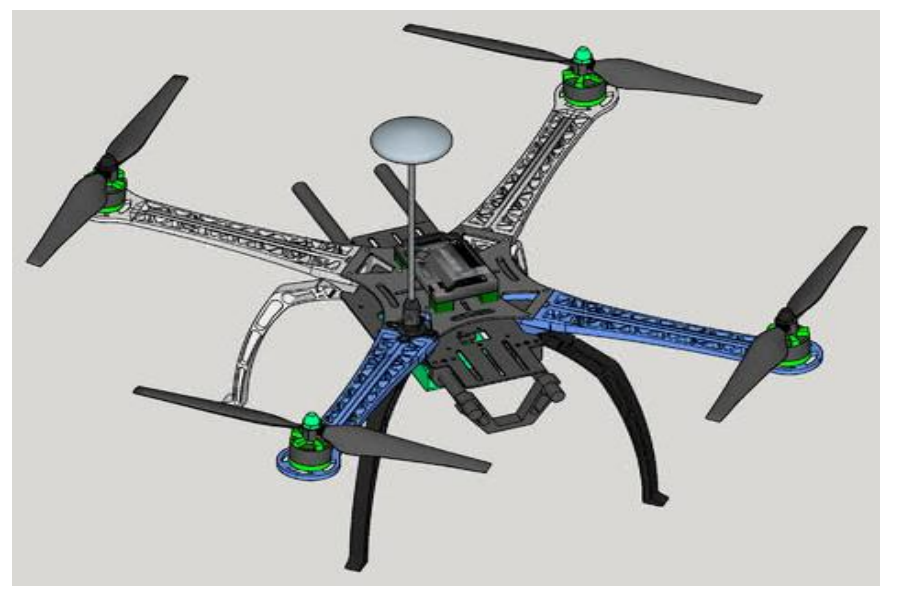

Fig. 1. "3D" modeling of the drone 


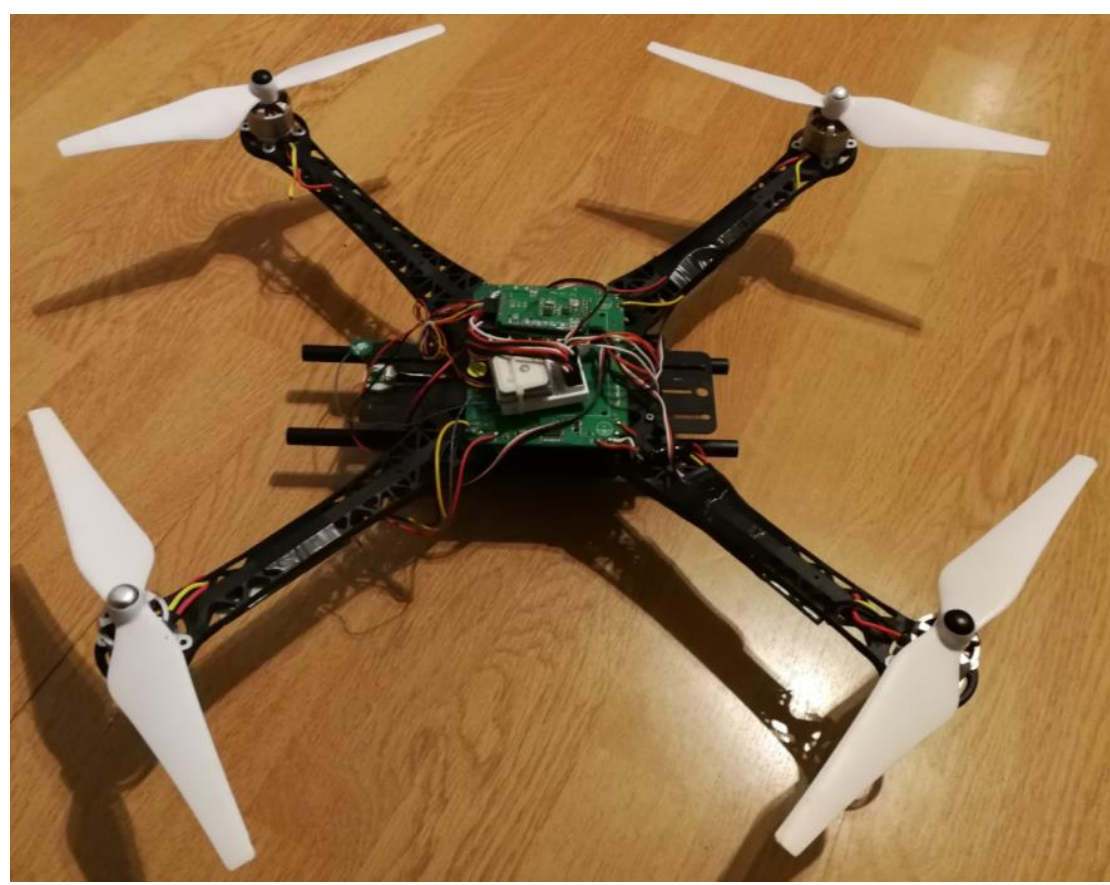

Fig. 2. Drone frame

\section{Monitoring of environmental parameters in contaminated sites with the help of drones}

The novelty of the technology developed within the project is the attempt to solve this lack of an intermediate resolution level by using the following methods of investigation:

- medium and low altitude aerial exploration performed using ground-based multi-rotor flight platforms;

- complex image processing with the help of advanced techniques in the field;

- digital models of high-resolution terrain;

- the extensive use of the most efficient techniques of geophysical investigation.

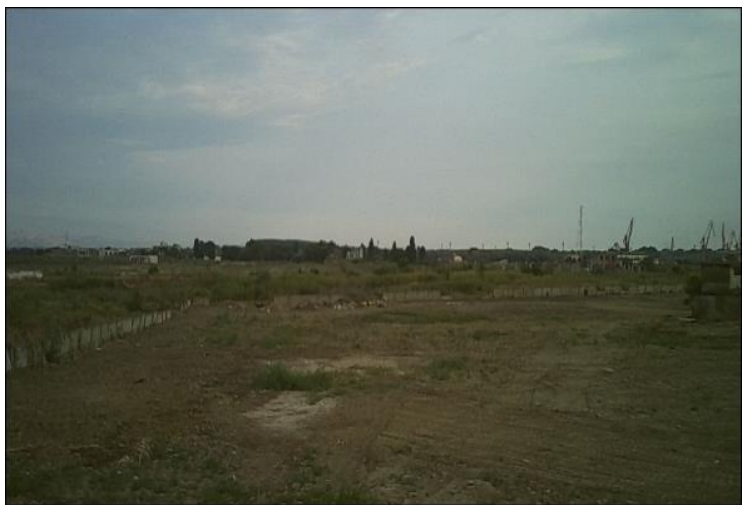

Fig. 3. Video testing on Braila-Galati dam
In the study of the monitoring of environmental parameters we used the drone together with a variety of sensors placed on different areas of the drone, which can perform a variety of monitoring tasks.

Ultrasonic sensors with a frequency of $44 \mathrm{KHz}$ were used to perform the acoustic monitoring, allowing them to record fine sounds.

In Figures 3 and 4 are video images taken from the Braila-Galati dam area, high resolution images that can show aspects needed for experimental research.

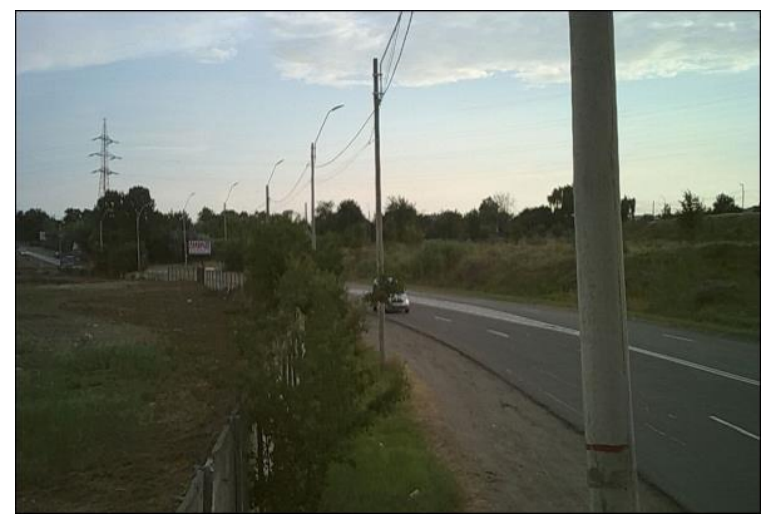

Fig. 4. Video testing on Braila-Galati dam

The digital camera allows video recording and image capture on a digital storage memory.

The digital camera of the drone has the following specifications:

- Sensor 12 Mega Pixel; 
-1.5-inch LCD display;

- Fast image processor;

- Photo resolution 12 sqm;

- HD video resolution 1920 x 1080/30 frames per second;

- $900 \mathrm{mAh}$ removable battery;

- Dimensions 29.8 x 59.2 x $41 \mathrm{~mm}$;

- Weight with battery $44 \mathrm{~g}$;

- Weight without battery $58 \mathrm{~g}$.

In Figure 5 is shown how depth measurements were performed and water samples were collected in August of 2019.

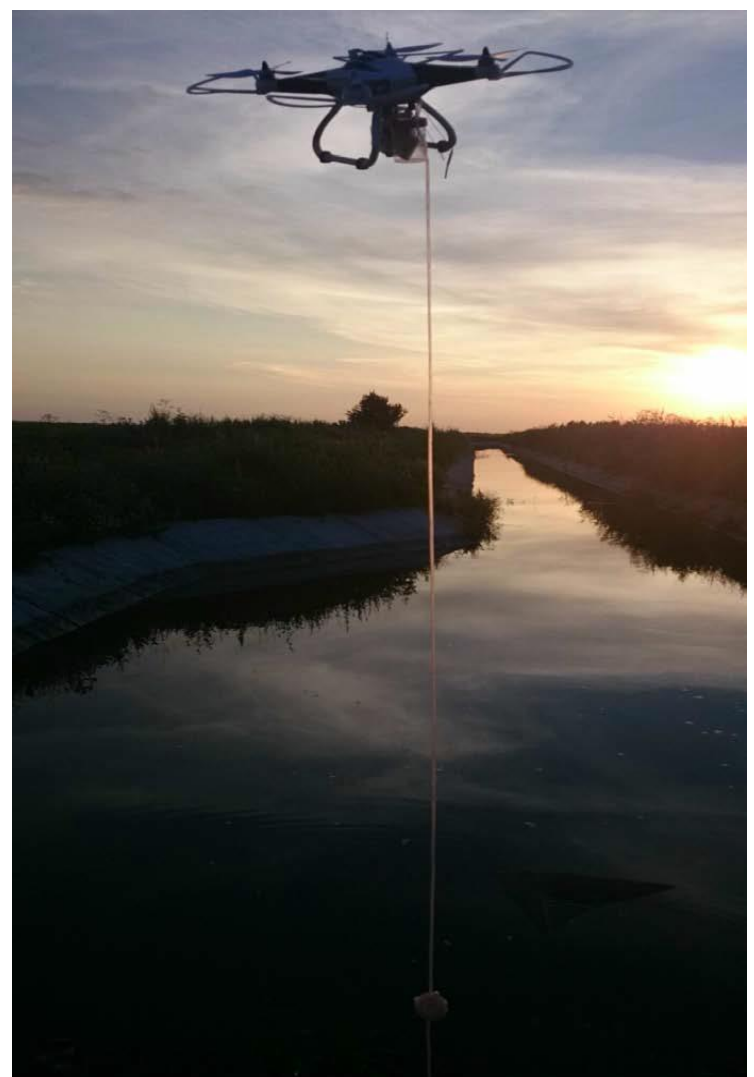

Fig. 5. Collecting water evidence

The determinations made in the laboratory of the water samples taken are presented in Table 1.

Liquid sampling was performed using the complex water extraction system. The following components have been fitted to perform the extraction of liquids from hard-to-reach areas:

- hose $3 \mathrm{~m}$ long;

- mini pump with electric drive;

- liquid sensor.

Laboratory measurements show small exceeding of the maximum allowed values

In Figure 6 is shown how were made determinations regarding the monitoring of the noxious at the crossroad between Călărași street and Griviței street in Brăila in 2019 (Table 2).
The values obtained were processed in laboratory and there are close to maximum values allowed.

Table 1. The values obtained from the collected samples

\begin{tabular}{|c|c|c|c|c|}
\hline & $\mathrm{pH}$ & $\begin{array}{c}\text { Dry } \\
\text { filterable } \\
\text { residue } \\
105^{\circ} \mathrm{C} \\
\mathrm{mg} / \mathrm{dm}^{3}\end{array}$ & $\begin{array}{c}\text { Total } \\
\mathrm{Fe} \\
\left(\mathrm{Fe}^{3+}\right) \\
\mathrm{mg} / \mathrm{dm}^{3}\end{array}$ & $\begin{array}{c}\text { Nitrates } \\
\left(\mathrm{NO}_{3}{ }^{-}\right) \\
\mathrm{mg} / \mathrm{dm}^{3}\end{array}$ \\
\hline $\begin{array}{c}\text { Normal } \\
\text { values }\end{array}$ & 8.5 & 1000 & 1 & 30 \\
\hline $\begin{array}{c}\text { Obtain } \\
\text { values }\end{array}$ & 8.6 & 1100 & 0.9 & 35 \\
\hline
\end{tabular}

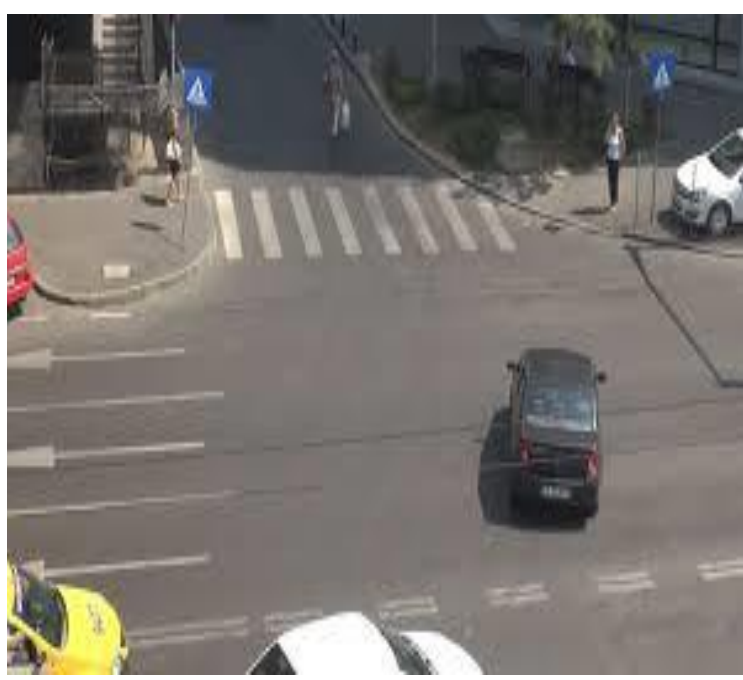

Fig. 6. Determination of traffic noxious

Table 2. Values obtained from research on pollution from intersections

\begin{tabular}{|c|c|c|c|}
\hline Month & $\begin{array}{c}\mathrm{CO} \\
\mu \mathrm{g} / \mathrm{m}^{3}\end{array}$ & $\begin{array}{c}\mathrm{NO}_{2} \\
\mu \mathrm{g} / \mathrm{m}^{3}\end{array}$ & $\begin{array}{c}\mathrm{SO}_{2} \\
\mu \mathrm{g} / \mathrm{m}^{3}\end{array}$ \\
\hline $\begin{array}{c}\text { Maximum } \\
\text { allowed } \\
\text { values }\end{array}$ & 15 & 0.1 & 0.2 \\
\hline January & 9 & 0.03 & 0.1 \\
\hline May & 11 & 0.06 & 0.15 \\
\hline August & 13 & 0.08 & 0.2 \\
\hline
\end{tabular}

With the help of the sensor attached to the drone, the quality of the air and the quantity of the noises can be monitored, and the drone will transmit in real time the values obtained with the help of his sensor to the base. 


\section{THE ANNALS OF "DUNAREA DE JOS” UNIVERSITY OF GALATI \\ FASCICLE IX. METALLURGY AND MATERIALS SCIENCE \\ No. 3 - 2019, ISSN 2668-4748; e-ISSN 2668-4756 \\ Article DOI: https://doi.org/10.35219/mms.2019.3.05}

By building the drone and equipped with last generation technology, it was able to monitor and take samples under optimum conditions and could transmit real-time minimum in-flight data on samples taken.

The drone comes with state-of-the-art technology, namely a small, ultra-powerful Chip that allows the drone to capture high quality images and videos. Subsequently, the artificial intelligence system with which the drone is equipped, serves to detect, recognize and learn the scenarios of everyday life. Drone is a first in the industry, when advanced visual guidance systems were introduced as standard functionality. The product finds its applicability both industrially and commercially.

The culture monitoring is performed by a 3D camera with a very high-performance technology, this having the performance to be able to create a $3 \mathrm{D}$ map in real time to monitor the state of the soil / agriculture

Soil temperature monitoring: Drones with multispectral or thermal sensors can identify dry areas or flooded areas, being able to transmit the situation in real time to make a quick decision in saving the crop.

The sampling of water from water ponds is a dangerous activity, with a high degree of risk, usually carried out by the environmental personnel at the active places, developing a more innovative process for collecting water samples from flooded ponds, settling ponds, evaporation ponds and other bodies of water, safe from the dangerous risks associated with this task.

\section{Conclusions}

The technology has developed very rapidly and new, more efficient monitoring methods and reduced costs for timely information collection and communication have emerged.

Drones come with state-of-the-art technology, namely a small, ultra-high-performance Chip that allows drones to capture high-quality images and videos.

The drone is a first in the industry, being for the first time introduced advanced visual guidance systems as standard functionality. The product finds its applicability both industrially and commercially.

With the help of drones, we can get high resolution photos and videos, with the help of the remote accessed camera we can get a very clear image enlargement. Scientists dealing with volcanic eruptions study from a distance, using drone imaging technology to check the trajectory of the ash thrown. Sometimes scientists even engage in risky missions to collect evidence using drones from cracks created by earthquakes.

\section{References}

[1]. ***, High-End solution CAD/CAM/CAE Siemens NX 8.0.

[2]. ***, http://www.digitaltrends.com/cool-tech/google-joinsnasa-and-others-to-work-on-drone-traffic-control-system/. 